\title{
Ginkgo biloba e memória - revisão sistemática
}

\author{
Ginkgo biloba and memory - systematic review
}

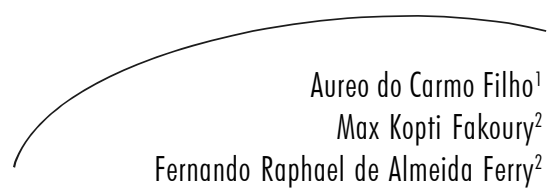

Resumo

Introdução: Com o envelhecimento da população, os distúrbios de memória têm-se tornado patologias cada vez mais frequentes. Com exceção de uma minoria dos casos cujas causas são reversíveis, estas doenças possuem prognóstico desanimador, curso inexorável e opções terapêuticas muito limitadas. O medicamento fitoterápico mais frequentemente utilizado para distúrbios de memória é o extrato seco de ginkgo biloba (GB). Suas ações antioxidantes, antiagregantes e vasodilatadoras têm encorajado diversos profissionais a utilizarem-no neste tratamento, porém não há registros convincentes que comprovem a eficácia do uso desta substância para tal fim. Objetivos: Avaliar a eficácia do GB na prevenção e no tratamento de distúrbios de memória. Métodos: Revisão sistemática da literatura dos últimos dez anos dos estudos clínicos duplocegos, randomizados, placebo-controlados, publicados na língua inglesa. Resultados e Conclusões: Embora tenhamos obtido poucos ensaios controlados sobre o tema, não há evidências suficientes para se indicar o uso da droga com a finalidade de tratar e/ou prevenir distúrbios de memória.

\section{Abstract}

Introduction: With the aging population, memory disorders have become more frequent diseases. Except for a minority of cases whose causes are reversible, these diseases have a dismal prognosis, inexorable course and very limited therapeutic options. The herbal medicine most often used for memory disorders is the dry extract of ginkgo biloba (GB). Its antioxidant, antiplatelet and vasodilatory actions have encouraged many professionals to use it in this treatment, but there are no convincing records showing the efficacy of this

Palavras-chave: Ginkgo Biloba. Transtornos da Memória. Memória. Distúrbios de Memória. Demência.

\footnotetext{
Hospital Universitário Gaffrée e Guinle. Rio de Janeiro, RJ, Brasil

Unidade de Terapia Intensiva

Departamento de Medicina Geral

Correspondência / Correspondence

Aureo do Carmo Filho

Hospital Universitário Gaffrée e Guinle. Unidade de Terapia Intensiva

Rua Mariz e Barros, 775 - Tijuca

20270-004 - Rio de Janeiro, RJ, Brasil

E-mail: aureocf@gmail.com
} 
substance for that purpose. Objectives: To evaluate the effectiveness of GB in the prevention and treatment of memory disorders. Methods: A systematic review of the literature of the last ten years of clinical double-blind, randomized, placebo-controlled, published in English. Results and Conclusions: Although we obtained few controlled trials on the subject, there is insufficient evidence for recommending the drug for the purpose of treating and / or prevention of memory disorders.
Key words: Ginkgo Biloba. Memory Disorders. Memory. Dementia.

\section{INTRODUÇÃO}

Ginkgo biloba (GB) é uma árvore nativa da China, Coreia e Japão, que foi considerada por Charles Darwin um "fóssil vivo". ${ }^{1} \mathrm{~A}$ árvore pode sobreviver por 1.000 anos e chegar a 30 metros de altura. É uma das espécies vegetais mais antigas do mundo, com relatos de prescrição de chás das folhas da árvore para fins medicinais que datam de 1436, durante a dinastia Ming. ${ }^{2}$ Preparações medicamentosas derivadas desta estão entre os medicamentos fitoterápicos mais prescritos no mundo, sendo utilizadas para uma série de doenças como problemas de concentração, tonteiras, zumbidos, cefaleias e particularmente, distúrbios cognitivos. ${ }^{2}$

Laboratórios farmacêuticos do mundo inteiro afirmam que o extrato de GB pode aumentar a concentração, melhorar a memória e aliviar sintomas de demência. Faltam, no entanto, estudos clínicos controlados que comprovem tal suposição. Mesmo com a carência de evidências científicas, cerca de 240 bilhões de dólares em produtos derivados do GB são vendidos nos Estados Unidos da América anualmente. ${ }^{2}$

O extrato de GB, denominado EGb761, contém porcentagens específicas de glicosídeos de ginkgoflavonas (24\%) e terpenóides (6\%), entre estes últimos os bilobalídeos e os ginkgolídeos A, B, C, M e J. ${ }^{3} \mathrm{~A}$ ação combinada dos diferentes princípios ativos presentes no extrato promove o incremento do suprimento sanguíneo cerebral pela vasodilatação e redução da viscosidade do sangue, além de reduzir a densidade de radicais livres de oxigênio nos tecidos nervosos. ${ }^{3} \mathrm{O}$ ginkgolídeo $\mathrm{B}$ é antagonista do receptor do fator ativador de plaquetas (PAF), daí suas propriedades de antiagregação plaquetária. ${ }^{4}$ Contudo, os benefícios à saúde humana, em particular sobre a cognição, não foram totalmente estabelecidos.-9

Vários estudos clínicos têm sido realizados para estabelecer as reais propriedades do GB, mas ainda continua duvidosa sua real eficácia. ${ }^{5-9} \mathrm{O}$ objetivo desta revisão é investigar a eficácia e a segurança deste medicamento para o tratamento e a prevenção de distúrbios de memória.

\section{MÉTODO}

Foi realizada busca eletrônica, em julho de 2007, nas bases de dados PUBMED, BIBLIOTECA COCHRANE, LILACS e BIREME, utilizando os descritores MeSH "Ginkgo biloba" e "memory disorders". Foram utilizados, ainda, listas de referências de todos os artigos adquiridos. Foram incluídos estudos clínicos duplo-cego, randomizados, placebo-controlados, publicados na língua inglesa entre os anos de 1997 e2007. Foi aplicada a escala de JADAD (1996) para validação dos ensaios clínicos, mas sem caráter de exclusão da presente revisão. Os resultados foram tabuladose apresentados por distribuição de frequência (quadro 1). 
Quadro 1 - Estudos incluídos nesta revisão. Rio de Janeiro, RJ, 2009.

\begin{tabular}{|c|c|c|c|}
\hline ESTUDO & AMOSTRA & METODOLOGIA & RESULTADOS \\
\hline $\begin{array}{l}\text { Mazza et al; } \\
2006\end{array}$ & $\begin{array}{l}\text { Pacientes portadores de } \\
\text { demência pela Doença de } \\
\text { Alzheimer (DSM-IV) }\end{array}$ & $\begin{array}{l}\text { Alocação dos pacientes em } 3 \text { grupos: GB } \\
\text { 160mg/dia, Donepezil } 5 \mathrm{mg} / \text { dia ou placebo. } \\
\text { Excluídos: demências de outras causas, } \\
\text { doenças orgânicas graves, pseudodemência } \\
\text { e esquizofrenia. Testagem mensal com } \\
\text { MEEM, ICG e TSK. }\end{array}$ & $\begin{array}{l}\text { Total de } 76 \text { pacientes. Grupos } \\
\text { donepezil e GB melhor que o } \\
\text { placebo, após } 6 \text { meses de } \\
\text { tratamento, no ICG. MEEM e } \\
\text { TSK semelhantes nos } 3 \text { grupos ao } \\
\text { final do estudo. Não houve } \\
\text { diferença quanto a efeitos } \\
\text { colaterais entre os grupos. }\end{array}$ \\
\hline $\begin{array}{l}\text { Solomon et } \\
\text { al; } 2002\end{array}$ & $\begin{array}{l}\text { Voluntários com idad e } \\
\text { superior a } 60 \text { anos, com } \\
\text { MMSE > 26, sem } \\
\text { comorbidades. }\end{array}$ & $\begin{array}{l}\text { Separação em } 2 \text { grupos: GB } 120 \mathrm{mg} / \text { dia ou } \\
\text { placebo. Utilizou-se os seguintes testes } \\
\text { psicométricos no início e ao final de } 26 \\
\text { meses: EWIA-R, TAVC, EWM -R, RV, TS, } \\
\text { TFCC, TNB e QM. }\end{array}$ & $\begin{array}{l}\text { Total de } 203 \text { pacientes; não houve } \\
\text { diferença estatisticamente } \\
\text { significativa entre os grupos em } \\
\text { nenhum dos testes e nem em } \\
\text { reações adversas dos } \\
\text { medicamentos. }\end{array}$ \\
\hline $\begin{array}{l}\text { Carlson JJ et } \\
\text { al; } 2007\end{array}$ & $\begin{array}{l}\text { Pacientes com idade entre } \\
65 \text { e } 85 \text { anos, sem } \\
\text { demência, com MMSE > } \\
24 \text { e sem outras } \\
\text { comorbidades } \\
\text { importantes. }\end{array}$ & $\begin{array}{l}\text { Separação em } 2 \text { grupos: placebo x GB } \\
\text { 160mg/dia. Visitas mensais por } 4 \text { meses e } \\
\text { testes cognitivos no início e ao final de } 4 \\
\text { meses: AVB, AGVC, JLO, MMSE, AL, } \\
\text { MSD, Qualidade de vida SF-36 e avaliação } \\
\text { de atividade plaquetária. }\end{array}$ & $\begin{array}{l}\text { Total de } 78 \text { pacientes. Somente o } \\
\text { AL mostrou diferença } \\
\text { estatisticamente significativa entre } \\
\text { os grupos, favorecendo o grupo } \\
\text { placebo. Sem diferença entre os } \\
\text { grupos em efeitos colaterais. }\end{array}$ \\
\hline $\begin{array}{l}\text { Dongen Mv } \\
\text { et al; } 2003\end{array}$ & $\begin{array}{l}\text { Multicêntrico; pac. com } \\
\text { demência ou transtorno } \\
\text { cognitivo leve, idade ? } 50 \\
\text { anos. }\end{array}$ & $\begin{array}{l}\text { Separação em } 3 \text { grupos: Placebo, GB } \\
\text { 240mg/dia e GB 160mg/dia; aplicou-se os } \\
\text { testes TSK, CGI e EGAAVDN. Testagem } \\
\text { no início do estudo e ao final de } 24 \\
\text { semanas. }\end{array}$ & $\begin{array}{l}\text { Total de } 123 \text { pacientes. Nenhuma } \\
\text { diferença entre os grupos, inclusive } \\
\text { em reações adversas. }\end{array}$ \\
\hline $\begin{array}{l}\text { Lovera J et } \\
\text { al; } 2007\end{array}$ & $\begin{array}{l}\text { Pacientes portadores de } \\
\text { esclerose múltipla (EM), } \\
\text { de } 18 \text { a } 60 \text { anos de idade, } \\
\text { sem déficits cognitivos, } \\
\text { depressão, não } \\
\text { dependentes de cuidador e } \\
\text { sem crises de EM nos } \\
\text { últimos } 30 \text { dias. }\end{array}$ & $\begin{array}{l}\text { Separação em } 2 \text { grupos: placebo e GB } \\
\text { 240mg/dia por } 12 \text { semanas. Utilizou-se os } \\
\text { testes: PASAT, TAVC, AGVC, MSD e } \\
\text { UFOV. }\end{array}$ & $\begin{array}{l}39 \text { pacientes randomizados. } \\
\text { Nenhuma diferença } \\
\text { estatisticamente significativa entre } \\
\text { os grupos, nem quanto a efeitos } \\
\text { colaterais. }\end{array}$ \\
\hline $\begin{array}{l}\text { Le Bars PL } \\
\text { et al; } 1997\end{array}$ & $\begin{array}{l}\text { Pacientes com demência } \\
\text { moderada a grave, sem } \\
\text { outras condições clínicas } \\
\text { importantes, idade? } 45 .\end{array}$ & $\begin{array}{l}\text { Separação em } 2 \text { grupos: placebo ou GB } \\
120 \mathrm{mg} \text { /dia por } 52 \text { semanas. Testagem no } \\
\text { início do estudo e após 12, } 26 \text { e } 52 \text { semanas. } \\
\text { Testes psicométricos utilizados: ADAS - } \\
\text { Cog, ICG e IAGA. }\end{array}$ & $\begin{array}{l}236 \text { pacientes randomizados. } \\
\text { Diferença estatisticamente } \\
\text { significativa entre os grupos ao } \\
\text { final de } 52 \text { semanas no ADAS-Cog } \\
\text { e IAGA. Efeitos colaterais } \\
\text { semelhantes entre os grupos. }\end{array}$ \\
\hline $\begin{array}{l}\text { Winther K } \\
\text { et al; } 1998\end{array}$ & $\begin{array}{l}\text { Pacientes de } 58 \text { a } 92 \text { anos } \\
\text { de idade, com distúrbio } \\
\text { cognitivo leve a } \\
\text { moderado, sem outras } \\
\text { comorbidades limitantes. }\end{array}$ & $\begin{array}{l}\text { Randomização em } 3 \text { grupos: GB } 120 \mathrm{mg} / \mathrm{dia} \\
\text { (GB120), GB } 240 \mathrm{mg} \text { /dia e placebo. } \\
\text { Testagem no início do estudo e após } 1 \text { e } 3 \\
\text { meses. Testes utilizados: EWM-R, EAMAA } \\
\text { e EAMAC. }\end{array}$ & $\begin{array}{l}54 \text { pacientes randomizados; vís } \\
\text { importante de seleção, pois no } \\
\text { grupo GB120 o EWM-R basal era } \\
\text { menor que nos outros grupos. Ao } \\
\text { final do estudo, o grupo GB120 } \\
\text { apresentava diferença importante } \\
\text { no EWM-R. Sem diferenças em } \\
\text { reações adversas. }\end{array}$ \\
\hline $\begin{array}{l}\text { Elsabagh S } \\
\text { et al; } 2005\end{array}$ & $\begin{array}{l}\text { Mulheres pós-menopausa, } \\
\text { sem distúrbios cognitivos } \\
\text { e/ou uso de psicoativos. }\end{array}$ & $\begin{array}{l}\text { Randomização em } 2 \text { grupos: placebo ou } \\
\text { GB } 120 \mathrm{mg} / \text { dia. Testagem no início e após } 6 \\
\text { semanas. Utilizou-se os testes: EWM-R, } \\
\text { PASAT, EVA, SoC e IEDS. }\end{array}$ & $\begin{array}{l}96 \text { pacientes; não houve diferença } \\
\text { significativa em nenhum dos testes } \\
\text { e nem quanto a efeitos colaterais. }\end{array}$ \\
\hline $\begin{array}{l}\text { Hartley DE } \\
\text { et al; } 2003\end{array}$ & $\begin{array}{l}\text { Mulheres pós-menopausa } \\
\text { com idade entre } 53 \text { e } 65 \\
\text { anos, sem distúrbios } \\
\text { cognitivos. }\end{array}$ & $\begin{array}{l}\text { Randomização em } 2 \text { grupos: placebo e GB } \\
120 \mathrm{mg} \text { /dia, por } 7 \text { dias. Testagem antes e } \\
\text { depois do uso do medicamento, utilizando- } \\
\text { se: EWM-R, IEDS, PASAT e DMTS. }\end{array}$ & $\begin{array}{l}31 \text { pacientes alocados. DMTS, } \\
\text { IDED e PASAT favoráveis ao GB. } \\
\text { Efeitos colaterais semelhantes. }\end{array}$ \\
\hline $\begin{array}{l}\text { Elsabagh S } \\
\text { et al; } 2005\end{array}$ & $\begin{array}{l}\text { Jovens saudáveis de } 18 \text { a } \\
26 \text { anos de idade. }\end{array}$ & $\begin{array}{l}\text { Separação em } 2 \text { grupos: placebo e GB } \\
120 \mathrm{mg} / \text { dia; testagem com IEDS, SoC e } \\
\text { outros, no início do estudo e ao fi nal de } 6 \\
\text { semanas. }\end{array}$ & $\begin{array}{l}52 \text { pacientes; nenhuma diferença } \\
\text { entre os grupos, inclusive em } \\
\text { reações adversas. }\end{array}$ \\
\hline $\begin{array}{l}\text { Moulton PL } \\
\text { et al; } 2001\end{array}$ & $\begin{array}{l}\text { Pacientes jovens } \\
\text { saudáveis. }\end{array}$ & $\begin{array}{l}\text { Separação em } 2 \text { grupos: placebo e GB } \\
120 \mathrm{mg} / \text { dia por } 5 \text { dias; testagem antes e } \\
\text { depois deste período, utilizando EWM-R, } \\
\text { Sternberg Memory Scan Test e Dareman } \\
\text { and Carpenter Test. }\end{array}$ & $\begin{array}{l}60 \text { pacientes; nenhuma diferença } \\
\text { estatisticamente significativa entre } \\
\text { os grupos. }\end{array}$ \\
\hline $\begin{array}{l}\text { Maurer K et } \\
\text { al; } 1997\end{array}$ & $\begin{array}{l}\text { Pacientes de } 50 \text { a } 80 \text { anos } \\
\text { de idade, com demência } \\
\text { leve a moderada, por } \\
\text { Doença de Alzheimer. }\end{array}$ & $\begin{array}{l}\text { Separação em } 2 \text { grupos: placebo e GB } \\
120 \text { mg/dia. Acompanhamento por } 3 \text { meses, } \\
\text { com testagem no início e ao final do } \\
\text { mesmo, utilizando os seguintes testes: TSK, } \\
\text { ADAS, ICG, Trailmaking e Multiple } \\
\text { Choice Vocabula ry Test. }\end{array}$ & $\begin{array}{l}18 \text { pacientes; grupos diferentes } \\
\text { (TSK no grupo placebo foi menor } \\
\text { no início do estudo). ICG } \\
\text { favorecendo o grupo GB no final } \\
\text { do estudo. Reações adversas } \\
\text { semelhantes. }\end{array}$ \\
\hline
\end{tabular}


A maioria dos estudos (66,67\%) foi feita com pacientes com idade superior a 45 anos e $33,33 \%$ analisaram pacientes mais jovens (idade igual ou superior a 18 anos). Em relação ao gênero, observamos que $44,30 \%$ da população da amostra é do sexo masculino e 55,70\% do sexo feminino. $41,67 \%$ dos trabalhos foram realizados com pacientes portadores de demência e/ ou transtorno cognitivo leve, sendo os outros trabalhos realizados com indivíduos sem qualquer déficit cognitivo.

A dose utilizada nos trabalhos variou de 120 a $240 \mathrm{mg} /$ dia, o que é exatamente a faixa terapêutica preconizada. ${ }^{2}$ Participaram do estudo tanto pacientes sem qualquer problema de memória ${ }^{11,12,15,18-21}$ quanto pacientes com demência e/ou distúrbios cognitivos leves. . $^{10,13,14,16,17,22}$

Mazza et al. ${ }^{10}$ procederam com a alocação de indivíduos com idade entre 50 e 80 anos, portadores de demência por doença de Alzheimer em três grupos distintos, sendo o primeiro grupo composto por pacientes que usaram $160 \mathrm{mg}$ de GB por dia; o segundo, $5 \mathrm{mg}$ de donepezil por dia, e o terceiro, placebo. Neste trabalho foram excluídos os pacientes com demência por outras causas, doenças orgânicas graves, pseudodemência e esquizofrenia. Os pacientes foram testados mensalmente com o Mini-Exame do Estado Mental (MEEM), Impressão Clínica Global (ICG) e Teste Syndrom-Kurz (TSK).

No trabalho de Solomon et al. ${ }^{11}$ foram estudados voluntários com idade superior a 60 anos sem comorbidades, com escore superior a 26 no MEEM de triagem. Os pacientes foram separados em dois grupos que usaram $120 \mathrm{mg}$ de GB por dia ou placebo. Realizaram-se os seguintes testes psicométricos no início e ao final de seis semanas: Escala Wechsler de Inteligência do Adulto Revisada (EWIA-R), Teste de Aprendizado Verbal da Califórnia (TAVC), Escala Wechsler de Memória Revisada (EWM-R), Reprodução Visual (RV), Teste Stroop (TS), Teste de Fluência de Categoria Controlada (TFCC), Teste de Nomeação de Boston (TNB) e Questionário de Memória (QM).

Carlson et al. ${ }^{12}$ estudaram pacientes sem demência e/ou outras comorbidades importantes, com idade entre 65 e 85 anos, que obtiveram pontuação maior que 24 no MEEM de triagem. Estes foram separados em dois grupos (placebo ou GB $160 \mathrm{mg} / \mathrm{dia}$ ), sendo testados mensalmente durante quatro meses, com as seguintes ferramentas: Aprendizado Visual de Benton (AVB), Associação Gráfica-Visual Controlada (AGVC), Julgamento de Linha de Orientação (JLO), MEEM, Aprendizado de Lista (AL), Modalidades Símbolo-Dígitos (MSD), Qualidade de Vida SF-36 e avaliação de atividade plaquetária.

Dongen et al. ${ }^{13,14}$ realizaram estudo multicêntrico com indivíduos com idade igual ou superior a 50 anos, portadores de demência ou transtorno cognitivo leve. Os 123 pacientes do estudo foram separados em três grupos: Placebo, GB $240 \mathrm{mg} / \mathrm{dia}$ e GB160mg/dia. Aplicaram-se os testes TSK, ICG e a Escala Gerontopsicológica de Avaliação de Atividades da Vida Diária de Nurenberg no início do estudo e ao final de 24 semanas.

No estudo de Lovera et al., ${ }^{15}$ portadores de esclerose múltipla (EM) de 18 a 60 anos de idade, sem déficits cognitivos, depressão, não dependentes de cuidador e sem crises de EM nos últimos 30 dias foram separados em dois grupos, utilizando placebo ou GB 240mg/dia por 12 semanas. Aplicaram-se os testes Paced Auditory Addition Test (PASAT), TAVC, AGVC, MSD e Useful Field ou Fiew (UFOV) no início e ao final do período de acompanhamento.

Le Bars et al. ${ }^{16}$ estudaram pacientes com demência moderada a grave, sem outras condições clínicas importantes, com idade igual ou superior a 45 anos. A amostra foi separada em dois grupos (placebo ou GB120mg/dia), que foram testados no início do estudo e após 12, 26 e 52 semanas, com as seguintes ferramentas: Subescala Cognitiva da Escala de Avaliação da Doença de Alzheimer (ADAS-Cog), ICG e Instrumento de Avaliação Geriátrica por Acompanhantes (IAGA).

Pacientes de 58 a 92 anos de idade, portadores de distúrbio cognitivo leve a moderado e sem outras comorbidades limitantes, foram estudados por Winther et al... ${ }^{17}$ Os pacientes foram separados em três grupos: Placebo, GB 120mg/dia (GB120) 
e GB 240mg/dia (GB240). Aplicaram-se os seguintes testes neuropsicológicos, no início do estudo e após um e três meses: EWM-R, Escala de Avaliação de Memória Autoaplicável e Escala de Avaliação de Memória Aplicada a Cuidador (EAMAC).

Mulheres na pós-menopausa, sem distúrbios cognitivos e/ou uso de psicoativos, foram estudadas por Elsabagh et al.. ${ }^{18}$ As pacientes foram separadas em dois grupos (placebo e GB120mg/ dia) e avaliadas ao início e após seis semanas de acompanhamento. Utilizaram-se os seguintes testes: EWM-R, PASAT, Escalas Visuais Analógicas (EVA), Stockings of Cambridge (SoC) e Intra/Extra Dimensional Shift (IEDS).

Hartley et al. ${ }^{19}$ estudaram mulheres na pósmenopausa, com idade entre 53 e 65 anos, sem distúrbios cognitivos. As pacientes foram separadas em dois grupos (placebo e GB $120 \mathrm{mg} / \mathrm{dia}$ ) e acompanhadas por sete dias, sendo testadas no início do estudo e após o período de acompanhamento, com as seguintes ferramentas: EWM-R, IEDS, PASAT e Delayed Matching-to-Sample (DMTS).

Elsabagh et al. ${ }^{20}$ avaliaram mulheres jovens saudáveis com idade entre 18 e 26 anos, separando-as em dois grupos (placebo e GB $120 \mathrm{mg} / \mathrm{dia}$ ) e utilizando as ferramentas EWMR, PASAT, EVA, SoC e IEDS no início do estudo e ao final de seis semanas.

Pacientes jovens saudáveis foram estudados por Moulton et al. ${ }^{21}$ Os indivíduos foram separados em dois grupos (placebo e GB $120 \mathrm{mg} / \mathrm{dia}$ ) e acompanhados por cinco dias, sendo avaliados antes e depois deste período pelas ferramentas: EWM-R, Sternberg Memory Scan Teste Dareman and Carpenter Test.

Maurer et al. ${ }^{22}$ estudaram pacientes de 50 a 80 anos de idade, com demência leve a moderada por doença de Alzheimer. Os pacientes foram separados em dois grupos (placebo e GB 120mg/ dia) e acompanhados por três meses, sendo avaliados no início e ao final deste período com as seguintes ferramentas: TSK, ADAS, ICG, Trailmaking e Multiple Choice Vocabulary Test.

\section{RESULTADOS}

De um total de 15 artigos obtidos nesta pesquisa, 12 foram selecionados para tabulação (quadro 1), uma vez que somente estes apresentaram uma metodologia organizada com critérios metodológicos rígidos e testes estatísticos adequados. Dos 12 trabalhos analisados, 41,67\% atingiram a escala de JADAD e 58,33\% não obtiveram o escore mínimo desejável para validação do trabalho (JADAD > 2).

No trabalho de Mazza et al., ${ }^{10} 150$ pacientes foram avaliados e, destes, somente 76 foram incluídos em três grupos: 25 pacientes usaram GB 160mg/dia; 25, donepezil 5mg/dia; e 26, placebo. Durante o acompanhamento, houve 15 abandonos, sendo cinco no grupo GB, seis no placebo e quatro no donepezil. Ao final de seis meses de acompanhamento, os pacientes que usaram donepezil e GB obtiveram melhor escore no ICG que o grupo placebo; o MEEM e o TSK tiveram escores semelhantes em todos os grupos.

Solomon et al. ${ }^{11}$ avaliaram inicialmente 338 pacientes, dos quais 230 preencheram os critérios de inclusão e foram separados em dois grupos (GB $120 \mathrm{mg} /$ dia e placebo). O grupo que usou placebo foi composto inicialmente por 115 pacientes e teve 16 abandonos ao longo do estudo. O grupo GB contou com 115 pacientes no início do acompanhamento e teve 11 abandonos. Não houve nenhuma diferença significativa entre os grupos nem ao início e nem ao final das seis semanas de acompanhamento, em nenhum dos testes psicométricos utilizados.

Noventa pacientes foram inicialmente estudados por Carlson et al.. ${ }^{12}$ Estes foram separados em dois grupos, sendo 46 alocados no grupo placebo e 44 no GB (160mg/dia). Ao final do acompanhamento, o grupo placebo tinha 36 pacientes e o GB tinha 42. O grupo que fez uso de placebo mostrou-se com escores mais altos no AL que o grupo GB ao final do estudo. Não houve diferença significativa entre os grupos nos outros testes, nem no início e nem ao final do período de quatro meses. 
Dongen et al. ${ }^{13}$ concluíram o estudo com 123 pacientes, sendo 44 no grupo placebo, 39 no grupo GB $240 \mathrm{mg} / \mathrm{dia}$ e 40 no grupo GB $160 \mathrm{mg} / \mathrm{dia}$. Ao longo do estudo, houve abandono de quatro pacientes do grupo placebo, quatro do GB $240 \mathrm{mg} /$ dia e um do GB 160mg/dia. Não se observou nenhuma diferença estatisticamente significativa entre os grupos em nenhuma das avaliações neuropsicológicas do estudo (no início do estudo e após 24 semanas de acompanhamento).

O estudo de Lovera et al. ${ }^{15} \mathrm{fez}$ inicialmente a triagem de 68 pacientes, dos quais 43 preencheram todos os critérios de inclusão, sendo então separados em dois grupos: 21 pacientes usaram GB $120 \mathrm{mg} /$ dia e 22 pacientes usaram placebo. Durante o mesmo, houve exclusão de um paciente do grupo GB e três pacientes do grupo placebo. Não houve qualquer diferença significativa entre os grupos em avaliações cognitivas no início do estudo e ao final do período de acompanhamento.

Le Bars et al. ${ }^{16}$ iniciaram o estudo com 327 pacientes (166 no grupo GB e 161 no grupo placebo). Ao final de 52 semanas de acompanhamento, os grupos eram compostos por 78 e 59 pacientes, respectivamente. $\mathrm{Na}$ avaliação final, observou-se diferença estatisticamente significativa em favor do grupo que utilizou GB nos escores ADAS-Cog e IAGA.

Winther et al. ${ }^{17}$ incluíram 54 pacientes no estudo (19 no grupo placebo, 19 no GB120 e 16 no GB240) e obtiveram diferença em favor do grupo que usou $G B$ no final do acompanhamento somente em um dos testes (EWM-R); entretanto, como os próprios autores relataram, não se pode atribuir tal diferença ao uso do medicamento, já que os grupos no início do estudo já apresentavam diferença significativa neste escore na testagem inicial.

Elsabagh et al. ${ }^{18}$ randomizaram 96 pacientes em seu estudo (48 no grupo GB e 48 no placebo), concluíram o acompanhamento com 87 pacientes (45 GB e 39 placebo) e não observaram qualquer diferençca significativa entre os grupos ao final do acompanhamento.
Hartley et al. ${ }^{19}$ alocaram 31 pacientes (16 no grupo placebo e 15 no GB). Ao final de sete dias, observaram diferença estatisticamente significativa em favor do grupo que usou GB nos testes DMTS, IDED e PASAT.

No estudo de Elsabagh et al., ${ }^{20} 52$ pacientes foram estudados (26 em cada grupo) e não houve nenhuma diferença entre os grupos ao final do acompanhamento.

Nenhuma diferença estatisticamente significativa foi observada no trabalho de Moulton et al., ${ }^{21}$ que estudou 60 pacientes $(30 \mathrm{em}$ cada grupo).

Maurer et al. ${ }^{22}$ estudaram 18 pacientes $(9 \mathrm{em}$ cada grupo) e observaram diferença significativa em favor do grupo que usou GB no ICG, não observando diferença nos testes neuropsicológicos avaliados.

\section{DISCUSSÃO}

Embora seja amplamente utilizada para melhorar a memória de pacientes com distúrbios cognitivos e/ou demência, os estudos realizados com estes pacientes não mostraram efeito benéfico irrefutável da droga quando comparado ao placebo. ${ }^{10,13,16,17,22,23}$ Nos trabalhos de Mazza et al. ${ }^{10}$ e Dongen et al., ${ }^{13}$ nenhuma diferença entre os grupos foi observada em testes neuropsicológicos no início do estudo e ao final de seis meses de tratamento.

Por outro lado, Le Bars et al. ${ }^{16}$ relataram diferença significativa entre os pacientes que usaram GB e placebo ao final de 52 semanas. Entretanto não foi descrito o método de randomização e o trabalho teve perdas importantes e assimétricas de pacientes entre os grupos, o que provavelmente pode ter interferido nos resultados: o grupo que usou o medicamento teve $50 \%$ e o grupo placebo, $72 \%$ de perdas.

Winther et al. ${ }^{17}$ e Maurer et al. ${ }^{22}$ relataram que o grupo de pacientes que utilizou GB ao final de três meses apresentou melhor desempenho em 
testes neuropsicológicos que os outros grupos; porém, estes grupos mostraram já uma diferença estatisticamente significativa nestes testes na avaliação inicial dos pacientes (antes do uso do medicamento), o que provavelmente pode ter interferido na análise, servindo como viés de seleção.

Muitas vezes, o GB é indicado para a prevenção de distúrbios de memória em indivíduos saudáveis. Nos estudos realizados com saudáveis, novamente não há evidências da eficácia do medicamento na melhora do desempenho em testes neuropsicológicos ${ }^{11,12,15,18,20} \mathrm{O}$ único estudo que relatou melhora em testes neuropsicológicos em pacientes que usaram GB foi o de Hartley et al.. ${ }^{19}$ No entanto, o estudo teve uma amostra pequena e apresentou um período de apenas uma semana para retestagem. Sabe-se que a retestagem neuropsicológica deve ser feita em período maior que duas semanas, pela tendência de melhores escores ocorrerem quando a mesma é realizada antes deste período. ${ }^{23}$

Todos os trabalhos mostraram ocorrência semelhante de reações adversas entre os pacientes que usaram GB ou placebo; há, porém, relatos na literatura que mostram que este medicamento pode aumentar a incidência de sangramentos importantes. . $^{24,25,26}$

Entre os trabalhos de melhor qualidade metodológica, somente o de Mazza et al. ${ }^{10}$ mostrou diferença significativa a favor do GB. No entanto, essa diferença só foi observada no escore de Impressão Clínica Global, enquanto que em testes psicométricos objetivos (MEEM, TSK) não houve qualquer diferença entre placebo, donepezil e GB.

Ressaltamos que o escore ICG não é específico para avaliação de memória. Estes estudos com JADAD > 2 englobaram 519 pacientes, sendo que apenas 76 pacientes concluíram o estudo de Mazza et al.. ${ }^{10}$ Esses trabalhos utilizaram um período mínimo de três meses para avaliar os efeitos da medicação, o que representa um tempo razoável para retestagem.

\section{CONCLUSÕES}

Em linhas gerais, não houve diferença significativa entre GB e placebo nas escalas de avaliação cognitiva. O uso de Ginkgo biloba parece ser seguro, pois a taxa de eventos adversos nos estudos descritos é similar à do placebo. Entretanto não há evidência suficiente que comprove a eficácia do medicamento no tratamento e/ou prevenção de distúrbios de memória.

Por outro lado, encontra-se em andamento um grande estudo multicêntrico ${ }^{27}$ em hospitais universitários dos Estados Unidos, realizado com idosos sem distúrbios cognitivos ou com transtornos cognitivos leves, que irá comparar o uso de GB $120 \mathrm{mg} /$ dia com placebo na prevenção da demência. O estudo está previsto para terminar em 2009 e poderá nos trazer mais algumas respostas. 


\section{REFERÊNCIAS}

1. Forlenza OV. Ginkgo biloba e memória: mito ou realidade? Rev Psiq Clín 2003; 30(6): 218-20.

2. DeFeudis FV. Ginkgo biloba extract (EGb 761): pharmacological activities and clinical applications. Elsevier 1991.

3. Kleijnen J, Knipschild P. Ginkgo biloba. Lancet 1992 nov; 340(8828): 1136-9.

4. Luo Y, et al. Inhibition of amyloid-beta aggregation and caspase- 3 activation by the Ginkgo biloba extract EGb 761. Proc Natl Acad Sci USA 2002 sep; 99(19): 12197-202.

5. Dartigues JF, et al. Vasodilators and nootropics as predictors of dementia and mortality in the PAQUID cohort. J Am Geriatr Soc 2007 mar; 55(3): 395-9.

6. Gabryelewicz T, Barcikowska M, Jarczewska DL. Alzheimer's disease therapy - theory and practice. Wiad Lek. 2005; 58(9-10): 528-35.

7. Frank B, Gupta S. A review of antioxidants and Alzheimer's disease. Ann Clin Psychiatry 2005 dec; 17(4): 269-86.

8. Pan HP. Progress in studies of pharmacological activities and clinical applications of preparations of dried leaf of Ginkgo biloba. Zhongguo Zhong Yao Za Zhi 2005 jan; 30(2): 93-6.

9. Kurz A, Van Baelen B. Ginkgo biloba compared with cholinesterase inhibitors in the treatment of dementia: a review based on meta-analyses by the Cochrane Collaboration. Dement Geriatr Cogn Disord 2004; 18(2): 217-26.

10. Mazza M, et al. Ginkgo biloba and donepezil: a comparison in the treatment of Alzheimer's dementia in a randomized placebo-controlled double-blind study. Eur J Neur 2006 sep; 13(9): 981-5.

11. Solomon PR, et al. Ginkgo for memory enhancement: a randomized controlled trial. JAMA 2002 ago; 288(7): 835-40.

12. Carlson JJ, et al. Safety and efficacy of a Ginkgo biloba-containing dietary supplement on cognitive function, quality of life, and platelet function in healthy, cognitively intact older adults. J Am Diet Assoc 2007 mar; 107(3): 422-32.

13. Van Dongen MC, et al. Ginkgo for elderly people with dementia and age-associated memory impairment: a randomized clinical trial. J Clin Epidemiol 2003 apr; 56(4): 367-76.

14. Van Dongen MC, et al. The efficacy of Ginkgo for elderly people with dementia and agerelated memory impairment: new results of a randomized clinical trial. J Am Geriatr Soc 2000 out; 48(10): 1183-94.

15. Lovera J, et al. Ginkgo biloba for the improvement of cognitive performance in multiple sclerosis: a randomized, placebocontrolled trial. Mult Scler 2007 apr; 13(3): 376-85.

16. Le Bars PL, et al. A placebo-controlled, doubleblind, randomized trial of an extract of Ginkgo biloba for dementia: north american Egb study group. JAMA 1997 out; 278(16): 1327-32.

17. Winther K, et al. Effects of Ginkgo biloba extract on cognitive function and blood pressure in elderly subjects. Curr Ther Res Clin Exp 1998; 59(12): 881-8.

18. Elsabagh S, Hartley DE, File SE. Limited cognitive benefits in stage +2 postmenopausal women after 6 weeks of treatment with Ginkgo biloba. J Psycopharmacol 2005 mar; 19(2): 173-81.

19. Hartley DE, et al. Effects on cognition and mood in postmenopausal women of 1-week treatment with Ginkgo biloba. Pharmacol Biochem Behav 2003 jun; 75(3): 711-20.

20. Elsabagh S, et al. Differential cognitive effects of Ginkgo biloba after acute and chronic treatment in healthy young volunteers. Psychopharmacology (Berl) 2005 may; 179(2): 437-46.

21. Moulton PL, et al. The effect of Ginkgo biloba on memory in healthy male volunteers. Physio Behav 2001 jul; 73(4): 659-65.

22. Maurer K, et al. Clinical efficacy of Ginkgo biloba special extract EGb 761 in dementia of the Alzheimer type. J Psychiat Res 1997 dec; 31(6): 645-55.

23. Chelune G, et al. Assessing reliable neuropsychological change: separating fact from fiction C.E. In: 25th Annual Meeting of the International Neuropsychological Society, 1997, Florida.

24. Schneider C, et al. Spontaneous hyphema caused by Ginkgo biloba extract. J Fr Ophtalmol 2002 sep; 25(7): 731-2.

25. MacVie OP, Harney BA. Vitreous haemorrhage associated with Gingko biloba use in a patient with age related macular disease. $\mathrm{Br} \mathrm{J}$ Ophthalmol 2005 oct; 89(10): 1378-9.

26. Yagmur E, et al. Bleeding complication under Gingko biloba medication. Am J Hematol 2005 aug; 79(4): 343-4.

27. Fitzpatrick AL, et al. Recruitment of the elderly into pharmacologic prevention trial: the Ginkgo Evaluation of Memory Study experience. Contemp Clin Trials 2006 dec; 27(6): 541-53. 